NISTIR 6465

\title{
NEW APPROACHES TO THE DEVELOPMENT OF FIRE-SAFE MATERIALS.
}

Alexander B. Morgan, Jeffrey W. Gilman, Marc Nyden, and Catheryn L. Jackson.

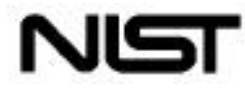

United States Department of Commerce

Technology Administration

National Institute of Standards and Technology 


\section{NEW APPROACHES TO THE DEVELOPMENT OF FIRE-SAFE MATERIALS.}

Alexander B. Morgan, Jeffrey W. Gilman, Marc Nyden

Building and Fire Research Laboratory

and Catheryn L. Jackson

Materials Science and Engineering Laboratory

National Institute of Standards and Technology

Gaithersburg, MD

February 2000

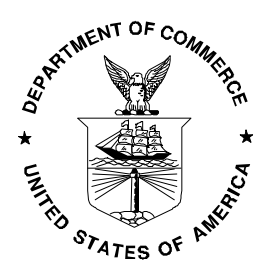

\section{U.S. Department of Commerce}

William M. Daley, Secretary

Technology Administration

Dr. Cheryl L. Shavers, Under Secretary of Commerce for Technology

National Institute of Standards and Technology

Raymond G. Kammer, Director 


\section{CONTENTS}

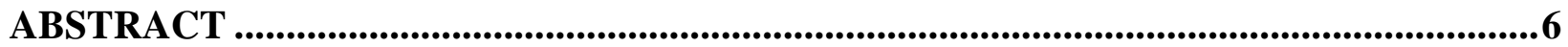

1.0 SYNTHESIS OF POLYETHERIMIDE-CLAY NANOCOMPOSITES. ........................7

1.1 Introduction............................................................................................................................................

1.2 Experimental Section. .............................................................................................................

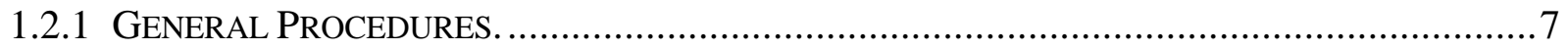

1.2.2 SyNTHESIS OF POLYETHERIMIDE 1-CLAY NANOCOMPOSITES...................................... 8

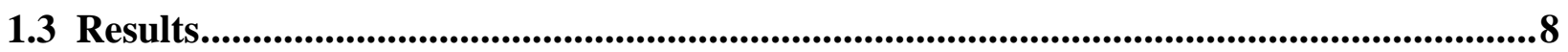

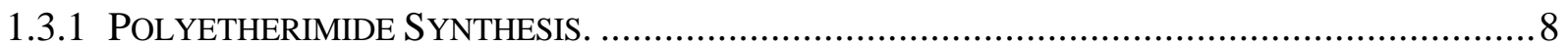

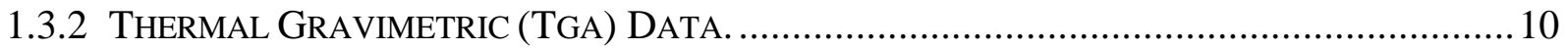

1.3.3 WIDE ANGLE X-RAY SCATTERING (WAXS) RESULTS................................................ 11

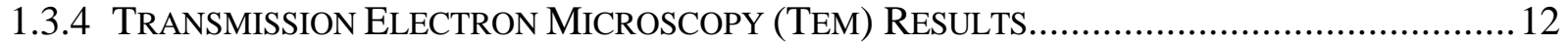

1.4 Conclusions On The Synthesis Of Polyetherimide-Clay Nanocomposites......................15

2.0 THERMALLY INDUCED CHANGES IN THE CONDENSED PHASE OF BURNING

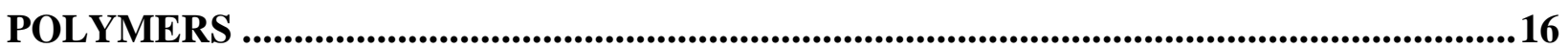

2.1 Introduction.......................................................................................................................................16

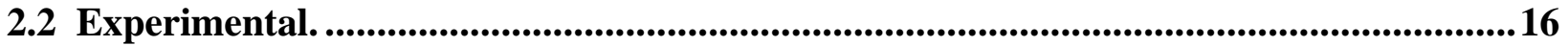

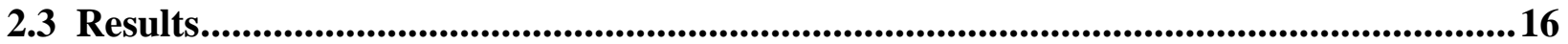

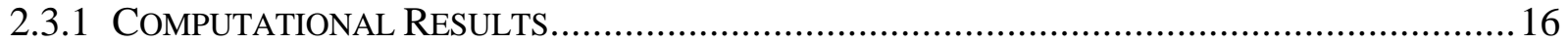

2.3.2 CONDENSED PHASE INFRARED ABSORBANCE RESULTS. ............................................. 17

2.4 Summary And Conclusions On Thermally Induced Changes In The Condensed Phase Of Burning Polymers. ..............................................................................................................................20

3. Acknowledgments ............................................................................................................................21

4. Future Work...........................................................................................................................................

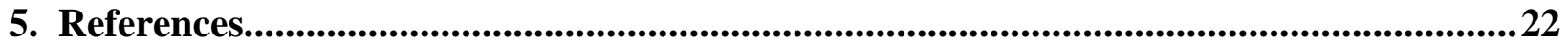




\section{LIST OF FIGURES}

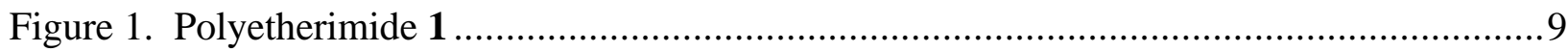

Figure 2. Tethering of PEI 1 to montmorillonite surface via 12-amino-1-dodecanoic acid

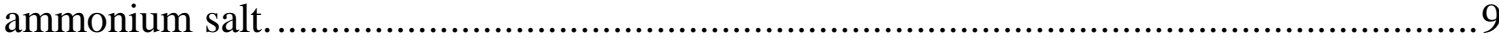

Figure 3. Synthesis of PEI 1-clay nanocomposites via in situ method..................................... 10

Figure 4. TGA curves for PEI 1, PEI 1+10wt\% MMT-C12, PEI 1+10wt\% MMT-C12Acid. Standard deviation for the wt $\%$ loss at $700{ }^{\circ} \mathrm{C}$ is: 0.25 for PEI 1, 0.49 for PEI $1+10 \mathrm{wt} \%$ MMT-C12, 0.62 for PEI 1+10wt\% MMT-C12Acid. TGAs were run under $\mathrm{N}_{2}$ and at 10 ${ }^{\circ} \mathrm{C} / \mathrm{min}$

Figure 5. TGA curves (derivative) of PEI 1, PEI 1+10wt\% MMT-C12, PEI 1+10wt\% MMTC12Acid. Standard deviation for the peak temperature is: 0.73 for PEI 1, 0.3 for PEI 1+10wt\% MMT-C12, 0.21 for PEI 1+10wt\% MMT-C12Acid. TGAs were run under $\mathrm{N}_{2}$ and at $10{ }^{\circ} \mathrm{C} / \mathrm{min}$

Figure 6. TGA curves of MMT-C12, MMT-C12Acid. Standard deviation for the wt $\%$ lost at $700{ }^{\circ} \mathrm{C}$ were: 1.04 for MMT-C12, 0.22 for MMT-C12Acid. TGAs were run under $\mathrm{N}_{2}$ at $10{ }^{\circ} \mathrm{C} / \mathrm{min}$

Figure 7. (A) WAXS plot of PEI 1 + 10wt\% MMT-C12 and MMT-C12 ............................... 12

Figure 8. (B) WAXS plot of PEI $1+10 \mathrm{wt} \%$ MMT-C12Acid and MMT-C12Acid. ...................12

Figure 9. (A) Low magnification picture (14000x) of Polymer $1+10 \mathrm{wt} \%$ MMT-C12. The darker lines (a) are clay and the white areas (b) are holes in the TEM section................13

Figure 10. (B) Medium magnification picture (140000x) of Polymer 1 + 10wt\% MMT-C12. Note single layers present $(\mathbf{a})$ as well as some multi-layer tactoids $(\mathbf{b}) \ldots \ldots \ldots \ldots \ldots \ldots \ldots \ldots \ldots . . . .13$

Figure 11. High magnification picture (600000x) of Polymer $1+10 \mathrm{wt} \%$ MMT-C12 showing a multi-layer tactoid. .......................................................................................... 13

Figure 12. (A) Low magnification picture (14000x) of Polymer $\mathbf{1}+10 \mathrm{wt} \%$ MMT-C12Acid. The small darker lines (a) are clay and the open white regions (b) are holes in the TEM section.................................................................................................... 14

Figure 13. (B) Medium magnification picture (140000x) of Polymer $1+10 \mathrm{wt} \%$ MMT-C12Acid. This is a domain with many multi-layer intercalated tactoids....................................... 14

Figure 14. High magnification picture (300000x) of Polymer $\mathbf{1}+10 \mathrm{wt} \%$ MMT-C12Acid. Some single layers (a) are present as well as multi-layer intercalated tactoids (b)................... 14

Figure 15. Computational data for polymer decompostions................................................... 17

Figure 16. Experimental set-up showing the fiber optic probe imbedded in the burning polymer.

Figure 17. The spectra obtained from nylon- 6 just before ignition (dashed line) and in the vicinity of the peak HRR (dotted line) are compared to a room temperature spectrum...19

Figure 18. The spectrum from nylon-6 (dashed line), which was measured just after ignition, is compared to the spectrum of the nylon-6/clay nanocomposite (solid line) measured about

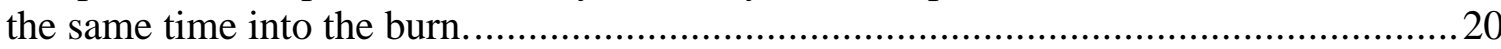

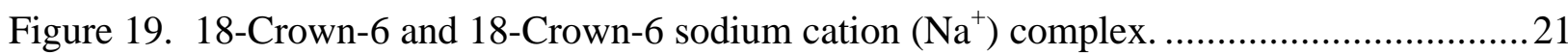




\title{
New Approaches to the Development of Fire-Safe Aircraft Materials
}

\author{
Alexander B. Morgan, Jeffrey W. Gilman, Marc R. Nyden \\ Building and Fire Research Laboratory \\ and Catheryn L. Jackson \\ Materials Science and Engineering Laboratory \\ National Institute of Standards and Technology, ${ }^{\dagger}$ Gaithersburg, MD 20899
}

\begin{abstract}
Thermoplastic polyetherimide-clay nanocomposites were synthesized from 1,3phenylenediamine and bisphenol A dianhydride using an in situ approach. Two types of organically treated clays were utilized to synthesize these nanocomposites. The two organically treated clays were montmorillonite clays treated with the ammonium salts of $n$-dodecylamine or 12-amino-1-dodecanoic acid. The dispersion of the clay in the polyetherimide was analyzed by wide-angle X-ray scattering and transmission electron microscopy. The results showed that the clay treated with the ammonium salt of 12-amino-1-dodecanoic acid gave a well-dispersed intercalated nanocomposite while the clay treated with the ammonium salt of $n$-dodecylamine gave a well-dispersed immiscible blend. These nanocomposites were then analyzed by thermal gravimetric analysis for their thermal stability.
\end{abstract}

Computational and experimental approaches were developed to explore the nature and consequences of thermally induced changes that occur in the condensed phase of burning polymers. A computational strategy for evaluating molecular weight distributions (which should enable us to calculate the melt viscosity during burning) from molecular dynamics simulations was developed based on a simple differential equation for the time dependence of the number average degree of polymerization, $x$, derived by Boyd. The experimental effort, which we hope to be able to use in validating the predictions of this model, was successful in obtaining real-time measurements of the mid-infrared spectra of burning polymers. The condensed phase spectra of nylon 6 and a nylon 6/clay nanocomposite were measured using a fiber optic reflectance probe while they were burning on the cone calorimeter.

\footnotetext{
${ }^{\dagger}$ This work was carried out by the National Institute of Standards and Technology (NIST), an agency of the U. S. government, and by statute is not subject to copyright in the United States.
} 


\subsection{SYNTHESIS OF POLYETHERIMIDE-CLAY NANOCOMPOSITES.}

\subsection{Introduction}

There is a desire to obtain zero heat-release rate (HRR) materials for aircraft interiors because a major area of concern in the airline industry is making aircraft safer and more crashsurvivable for passengers. A prime cause of death and injury during aircraft crashes is leakage of fuel into the fuselage, after which the fuel ignites, killing the passengers by flame or by asphyxiation from noxious fumes released. About $20 \%$ of all deaths on US airlines between 1981 and 1990 occurred in crash-survivable accidents where fire was created upon impact. ${ }^{1}$ It is further approximated that $40 \%$ of these deaths were caused by noxious fumes and toxic combustion products from the burning of various plastic or polymeric material present on the aircraft. ${ }^{1}$ The goal of our task within the Federal Aviation Administration (FAA) Interagency agreement program is to develop zero HRR materials. The candidate polymers that could reach this goal include polycarbonate and polyetherimides. Polymer clay nanocomposites have attracted a great deal of interest due to their improved mechanical, thermal, and flammability properties. $^{2}$ The improved flammability properties have led our laboratory to undertake a large research project in this area. ${ }^{3}$ Recently, reports on polyetherimide-clay nanocomposites ${ }^{4}$ led us to pursue the synthesis of a thermoplastic polyetherimide (PEI). However, these PEI systems are considered to be intractable, in that they are poorly soluble and their melting temperatures are so high, thus prohibitive to facile engineering or aircraft interior components. Therefore, a lowermelting PEI would be of great interest.

There are several ways to make polymer clay nanocomposites. Solution mixing, melt blending, and in situ polymerization can all yield polymer clay nanocomposites. 5 Obviously, solution mixing or melt blending are the easier routes towards making polymer clay nanocomposites. However, these routes can be limited by the solubility of the polymer and the dispersability of the clay. Using an organically treated clay will allow the clay to be more miscible with a polymer matrix or in a solvent. The main problem with these organic treatments is that their thermal stability limits their use in certain high melting polymers, such as aromatic PEIs. Therefore melt blending can only occur if the organic treatment on the clay can survive high temperature conditions. Currently there are no organic treatments which can survive typical processing temperatures for thermoplastic aromatic PEIs $\left(+350{ }^{\circ} \mathrm{C}\right)$. This leaves solution blending and in situ routes as the ones most likely to succeed in producing a PEI-clay nanocomposite. Our goal was to prepare a PEI treated clay ( $>50 \mathrm{wt} \%$ clay) that was stable above $350{ }^{\circ} \mathrm{C}$ and could be melt-blended with more PEI to prepare a $10 \mathrm{wt} \%$ clay PEI-clay nanocomposite. We have recently synthesized thermoplastic polyetherimide clay nanocomposites using an in situ polymerization, forming a polyamic acid-clay nanocomposite, which was then imidized to give the polyetherimide clay nanocomposite.

\subsection{Experimental Section.}

1.2.1 General Procedures.

All organic treated clays (sodium montmorillonite: Na-MMT, montmorillonite treated with n-dodecylamine ammonium salt: MMT-C12, montmorillonite treated with 12-

\footnotetext{
Certain commercial equipment, instruments, materials or companies are identified in this paper in order to adequately specify the experimental procedure. This in no way implies endorsement or recommendation by NIST.
} 
aminododecanoic acid ammonium salt: MMT-C12Acid) were provided by Southern Clay Products. These clays were dried for 2 hours at $110{ }^{\circ} \mathrm{C}$ in a forced air oven prior to use. N,N'Dimethylacetamide (DMAc) was purchased from Sigma-Aldrich Inc., distilled over $\mathrm{CaH}_{2}$, and stored over $3 \AA$ molecular sieves under $\mathrm{N}_{2}$ prior to use. Bisphenol A dianhydride (BPADA) and polyetherimide 1 were obtained from General Electric Corporation and contained 5wt $\%$ phthalic anhydride to control molecular weight of polymers using BPADA as a monomer. 1,3Phenylenediamine was purchased from Aldrich, vacuum distilled and stored under $\mathrm{N}_{2}$ prior to use. All other solvents were purchased from Acros Organics and were used as received. Thermal gravimetric analysis (TGA) data were collected on a SDT-2960 from TA Instruments. Wide angle X-ray scattering (WAXS) data was collected on a Phillips diffractometer using $\mathrm{Cu}$ $\mathrm{K} \alpha$ radiation, $(\lambda=0.1505945 \mathrm{~nm})$. The polyetherimide samples were ground to a particle size of less than $40 \mu \mathrm{m}$. Bright field transmission electron microscopy (TEM) images of polyetherimide 1/layered silicate (clay) nanocomposites were obtained at $120 \mathrm{kV}$, at low dose conditions, with a Phillips 400T electron microscope. The samples were ultramicrotomed with a diamond knife on a Leica Ultracut UCT microtome at room temperature to give $70 \mathrm{~nm}$ thick sections. The sections were transferred from water to carbon-coated (type B) $\mathrm{Cu}$ grids of 200 mesh. The contrast between the layered silicates and the polymer phase was sufficient for imaging, so no heavy metal staining of sections prior to imaging was required.

\subsubsection{Synthesis of Polyetherimide 1-Clay Nanocomposites.}

To a $250 \mathrm{~mL}$ round bottom flask equipped with a magnetic stirbar was added 1,3phenylenediamine $(0.77 \mathrm{~g}, 7.15 \mathrm{mmol})$. The flask was then sealed under a $\mathrm{N}_{2}$ atmosphere with a rubber septum. DMAc $(50 \mathrm{~mL})$ was then added and the solution was allowed to stir at R.T. for 1 hour. The septum was then removed, BPADA $(3.72 \mathrm{~g}, 7.15 \mathrm{mmol})$ was added quickly, and the septum was replaced. This reaction mixture was allowed to stir at R.T. for 1 hour. An organic treated clay (MMT-C12 or MMT-C12Acid, $0.5 \mathrm{~g}$ ) and $50 \mathrm{~mL}$ of DMAc were added to a separate $100 \mathrm{~mL}$ round bottom flask equipped with a magnetic stirbar. This mixture was heated to $90{ }^{\circ} \mathrm{C}$ for 5 hours to give a tan-colored translucent suspension. The septum on the flask containing the polyamic acid of polymer 1 was removed, and the MMT/DMAc suspension was added. The resulting polyamic acid/clay mixture was allowed to stir at R.T. overnight (approximately 12 hours). Upon completion of the reaction, the DMAc was removed via rotary evaporation and the resulting brown product was dried in vacuo. The material was then heated to $250{ }^{\circ} \mathrm{C}$ for 2 hours in a vacuum oven to give polyetherimide 1-clay nanocomposites.

\subsection{Results}

\subsubsection{Polyetherimide Synthesis.}

Several attempts were made to synthesize a polyetherimide-clay nanocomposite. Initially we tried using PEI 1 (Figure 1) and sodium montmorillonite (Na-MMT) via melt blending. We chose Na-MMT because of its high thermal stability, which would allow it to survive polyetherimide 1's typical processing conditions of $350{ }^{\circ} \mathrm{C}$. However, since Na-MMT has no organic treatment, it is highly immiscible in PEI 1, and no intercalation or exfoliation of the clay occurred using melt blending. WAXS was used to determine this result. Specifically, WAXS showed no change in the basal d-spacing for Na-MMT, indicating that no polymer had intercalated between the layers of the clay. We also tried solution blending of 1 and Na-MMT using methylene chloride, 1,3-dioxolane and N,N-dimethylacetamide (DMAc). WAXS showed that solvent intercalated, but no intercalation or exfoliation of $\mathbf{1}$ occurred. We then tried the in 
situ approach of making polymer clay nanocomposites, using two organically treated clays. Since DMAc is the solvent of choice for the polymerization of $\mathbf{1}$, we chose two organically treated clays which were shown by light scattering to disperse in DMAc. ${ }^{6}$ The two clays were montmorillonite treated with the ammonium salt of $n$-dodecylamine (MMT-C12) or the ammonium salt of 12-amino-1-dodecanoic acid (MMT-C12Acid). The MMT-C12Acid has the potential for reacting with the monomers during polymerization, creating a potential "tethered" polymer clay nanocomposite (Figure 2 ).

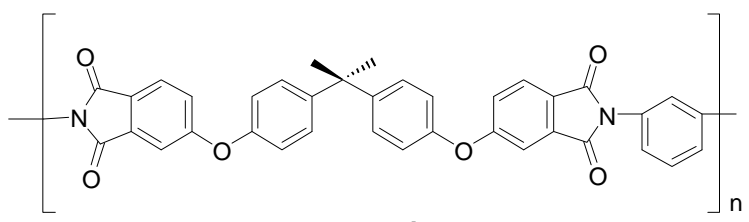

1

Figure 1. Polyetherimide 1

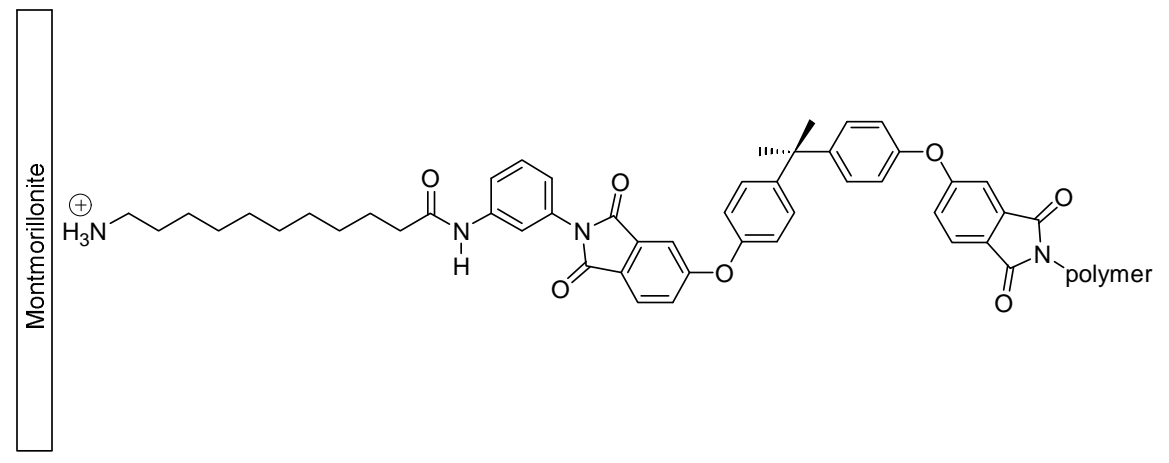

Figure 2. Tethering of PEI 1 to montmorillonite surface via 12-amino-1-dodecanoic acid ammonium salt.

We synthesized the polyetherimides from 1,3-phenylenediamine and bisphenol A dianhydride (BPADA) using DMAc as the solvent (Figure 3). The 1,3-phenylenediamine and BPADA was allowed to stir for 1 hour at room temperature to make a polyamic acid. The organically treated clay was then added to give a polyamic acid-clay nanocomposite. The solvent was then removed and the polyamic acid was imidized to the polyetherimide in a vacuum oven at $250{ }^{\circ} \mathrm{C}$ for 2 hours. The resulting material was then analyzed by thermal gravimetric analysis (TGA) for its thermal stability.

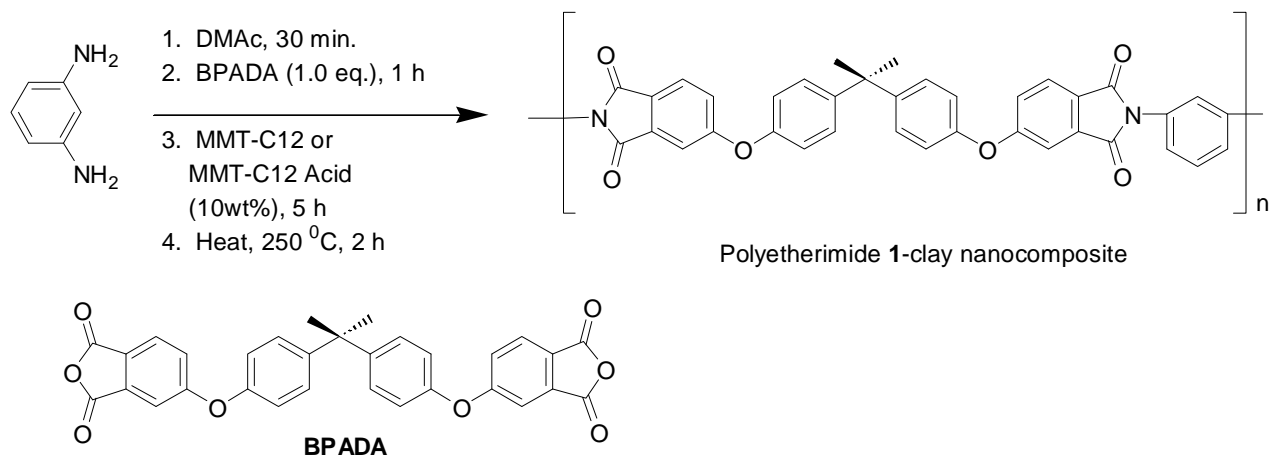


Figure 3. Synthesis of PEI 1-clay nanocomposites via in situ method.

\subsubsection{Thermal Gravimetric (TGA) data.}

Based on the above TGA data shown in Figures 4 and 5, it appears that the organic treatment on MMT-C12 and MMT-C12Acid begins to decompose at lower temperatures, resulting in a lower char yield for these nanocomposites. This is also reflected in the TGA data for MMT-C12 and MMT-C12Acid with no PEI present (Figure 6). There are some other potential reasons for these lower char yields. 1,3-Phenylenediamine, one of the necessary monomers for the synthesis, is very susceptible to oxidation. This can result in lower molecular weight polyetherimide and incomplete imidization, thus creating defects in the polymer chain. The current $\operatorname{method}^{7}$ used to synthesize polymer $\mathbf{1}$ is to use 1,3-phenylene bis(3-nitromaleimide) and the sodium salt of bisphenol A. These monomers are not susceptible to oxidation. This polymerization utilizes a nucleophilic addition reaction to facilitate polymerization which leads directly to polymer $\mathbf{1}$ rather than a polyamic acid. This route has an advantage over the polyamic acid route in that it does not have to undergo an imidization after initial polymerization, thus keeping the number of potential defects in the polymer backbone low.

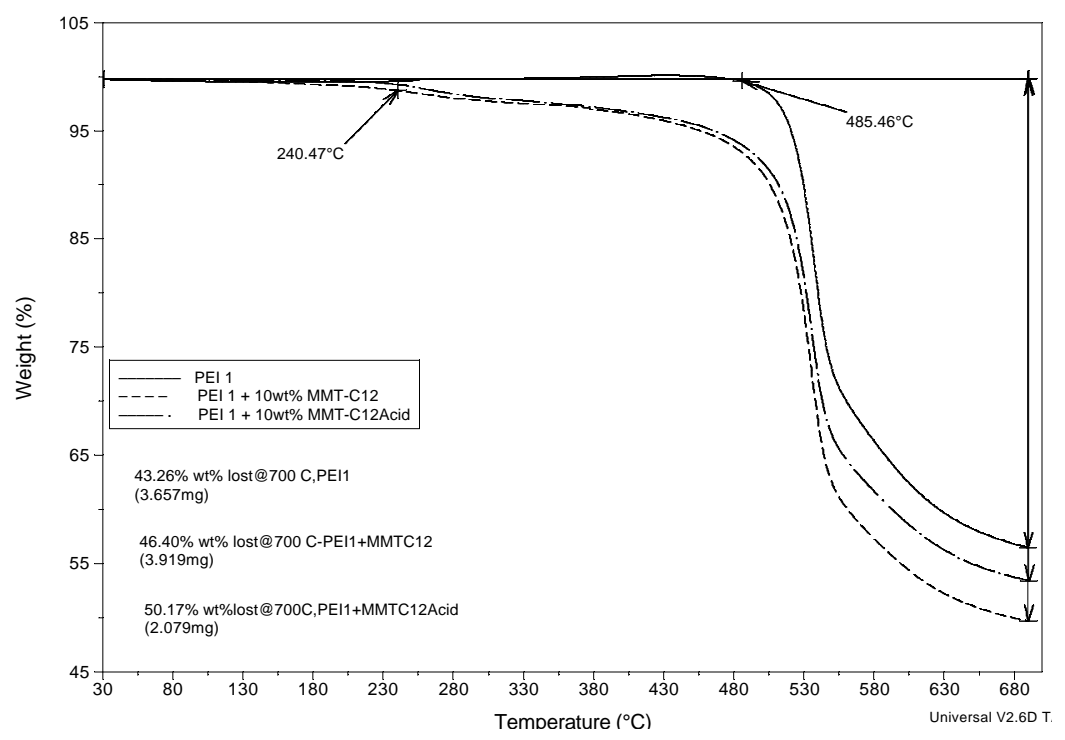

Figure 4. TGA curves for PEI 1, PEI 1+10wt\% MMT-C12, PEI 1+10wt\% MMT-C12Acid. Standard deviation for the wt $\%$ loss at $700{ }^{\circ} \mathrm{C}$ is: 0.25 for PEI 1, 0.49 for PEI $1+10 \mathrm{wt} \%$ MMTC12, 0.62 for PEI $1+10 \mathrm{wt} \%$ MMT-C12Acid. TGAs were run under $\mathrm{N}_{2}$ and at $10{ }^{\circ} \mathrm{C} / \mathrm{min}$. 


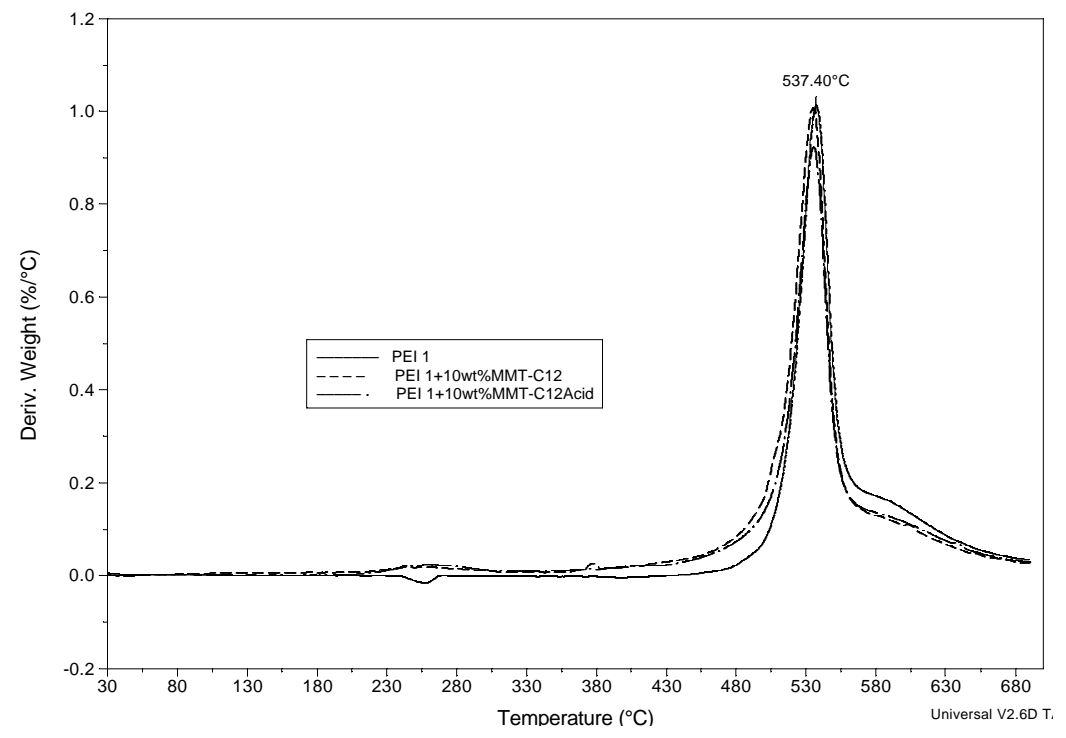

Figure 5. TGA curves (derivative) of PEI 1, PEI 1+10wt\% MMT-C12, PEI 1+10wt\% MMTC12Acid. Standard deviation for the peak temperature is: 0.73 for PEI 1, 0.3 for PEI 1+10wt $\%$ MMT-C12, 0.21 for PEI 1+10wt\% MMT-C12Acid. TGAs were run under $\mathrm{N}_{2}$ and at $10{ }^{\circ} \mathrm{C} / \mathrm{min}$.

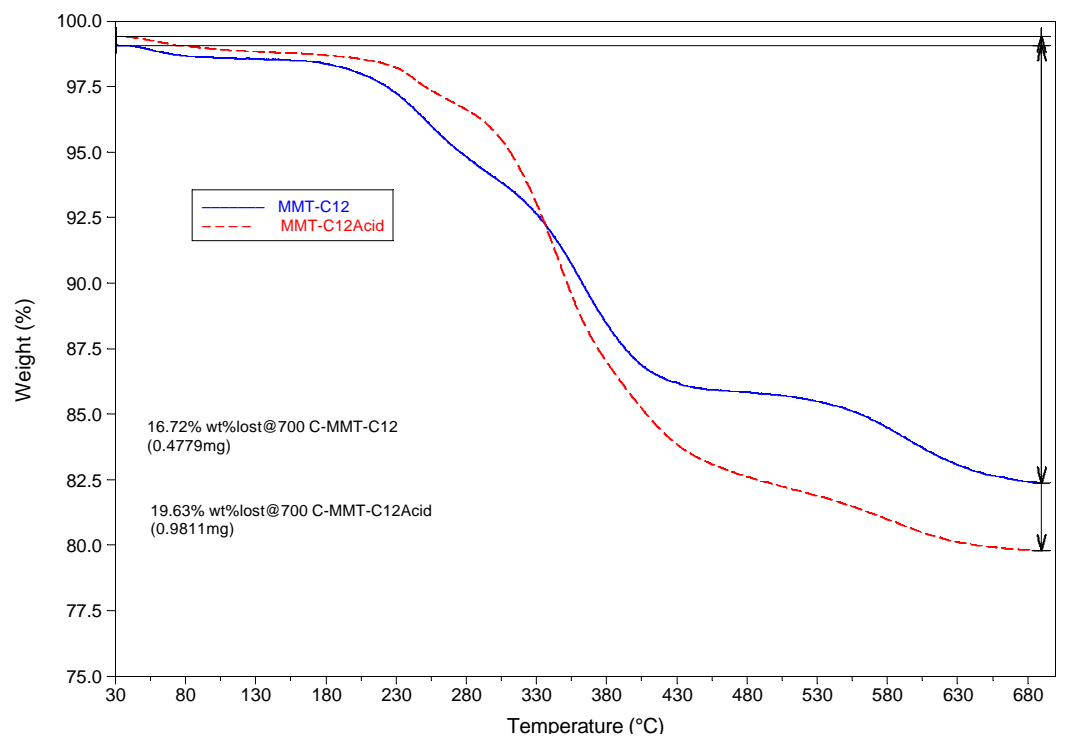

Figure 6. TGA curves of MMT-C12, MMT-C12Acid. Standard deviation for the wt $\%$ lost at $700{ }^{\circ} \mathrm{C}$ were: 1.04 for MMT-C12, 0.22 for MMT-C12Acid. TGAs were run under $\mathrm{N}_{2}$ at 10 ${ }^{\circ} \mathrm{C} / \mathrm{min}$.

\subsubsection{Wide Angle X-Ray Scattering (WAXS) Results.}

Using WAXS we investigated how well the clay was dispersed in PEI 1. WAXS plots are shown below (Figures 7-8). WAXS indicated that the d-spacing (basal distance between montmorillonite layers) in MMT-C12Acid increased from $1.36 \mathrm{~nm}$ to $1.92 \mathrm{~nm}$, following the in situ polymerization. This indicated that an intercalated PEI nanocomposite had been 
synthesized. However, no d-spacing change occurred when MMT-C12 was used during the synthesis of the polyetherimide. This would indicate that an immiscible blend had been synthesized. However, when these materials were examined by TEM we observed significant dispersion and exfoliation of some of the layered-silicate.
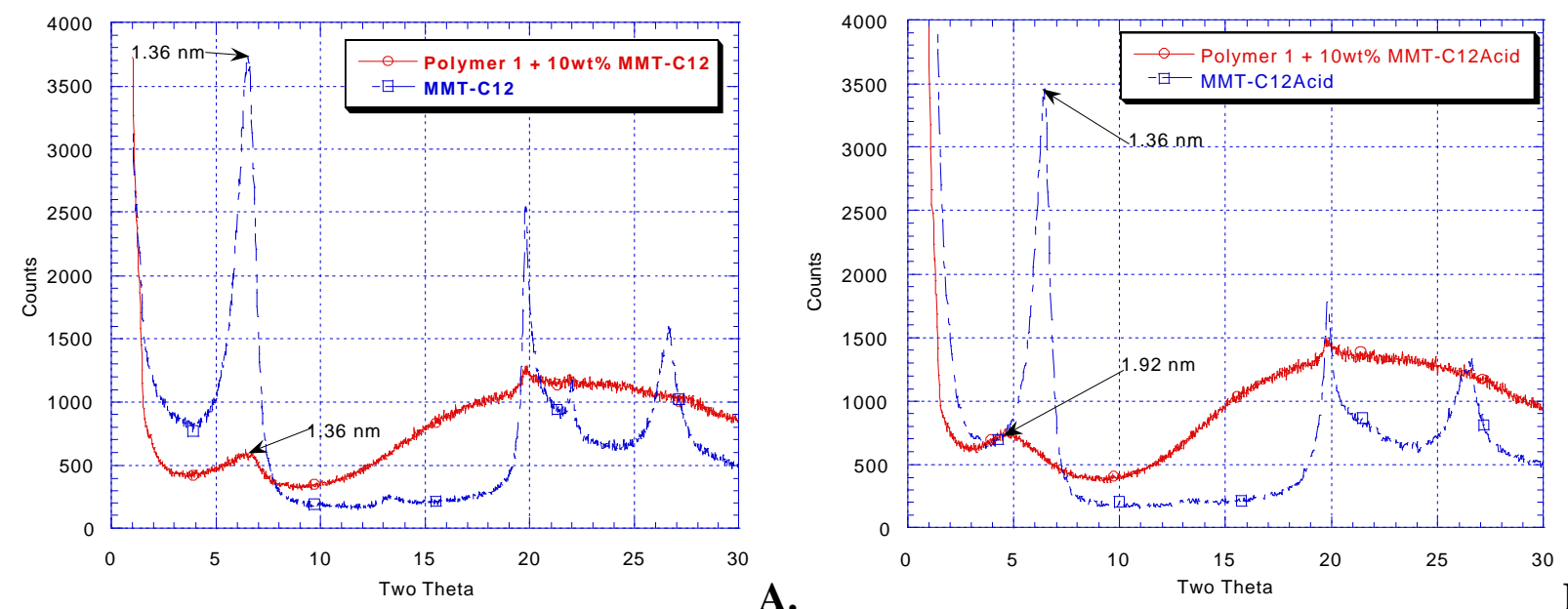

B.

Figure 7. (A) WAXS plot of PEI 1 + 10wt\% MMT-C12 and MMT-C12.

Figure 8. (B) WAXS plot of PEI 1 + 10wt\% MMT-C12Acid and MMT-C12Acid.

\subsubsection{Transmission Electron Microscopy (TEM) Results.}

TEM analysis tends to support these findings, but shows that the clay is well dispersed in both systems, including the immiscible MMT-C12/1 system. Some holes (white areas) are present in the microtomed thin films (Figure 9, 12); these are caused by knife damage during cutting and are not inherent in the nanocomposite material. In Figures 9-14, the darker lines are the clay layers and the polymer matrix is gray. TEM for MMT-C12/1 (Figure 9) shows that where multi-layer tactoids exist, the clay layers are tightly packed together, but there are no large, undispersed clay tactoids. A fair number of single layers were also observed (Figure 10). This indicates that the MMT-C12 was well dispersed and exfoliated in the DMAc before polymerization, but no polymer chains intercalated between the layers of this material during polymerization. Individual exfoliated layers in the solvent were encased in polymer, while multi-layer tactoids were similarly encased, leaving their original d-spacing intact. Thus, the polymerization occurred outside of the clay layers, giving the mixtures seen in figures 9-11. TEM of the MMT-C12Acid/1 system indicate that the clay was well dispersed, and some polymer intercalated in between the clay layers (Figures 12,13). Not as many single layers were seen in this system, but some did exist. The MMT-C12Acid/1 system is a mixed dispersion system, having some intercalated domains, but also some single layers, making it neither fully intercalated nor exfoliated. MMT-C12/1 also fits into this mixed dispersion category, as it has single layers indicating exfoliation, but multi-layer tactoids indicating immiscibility. 

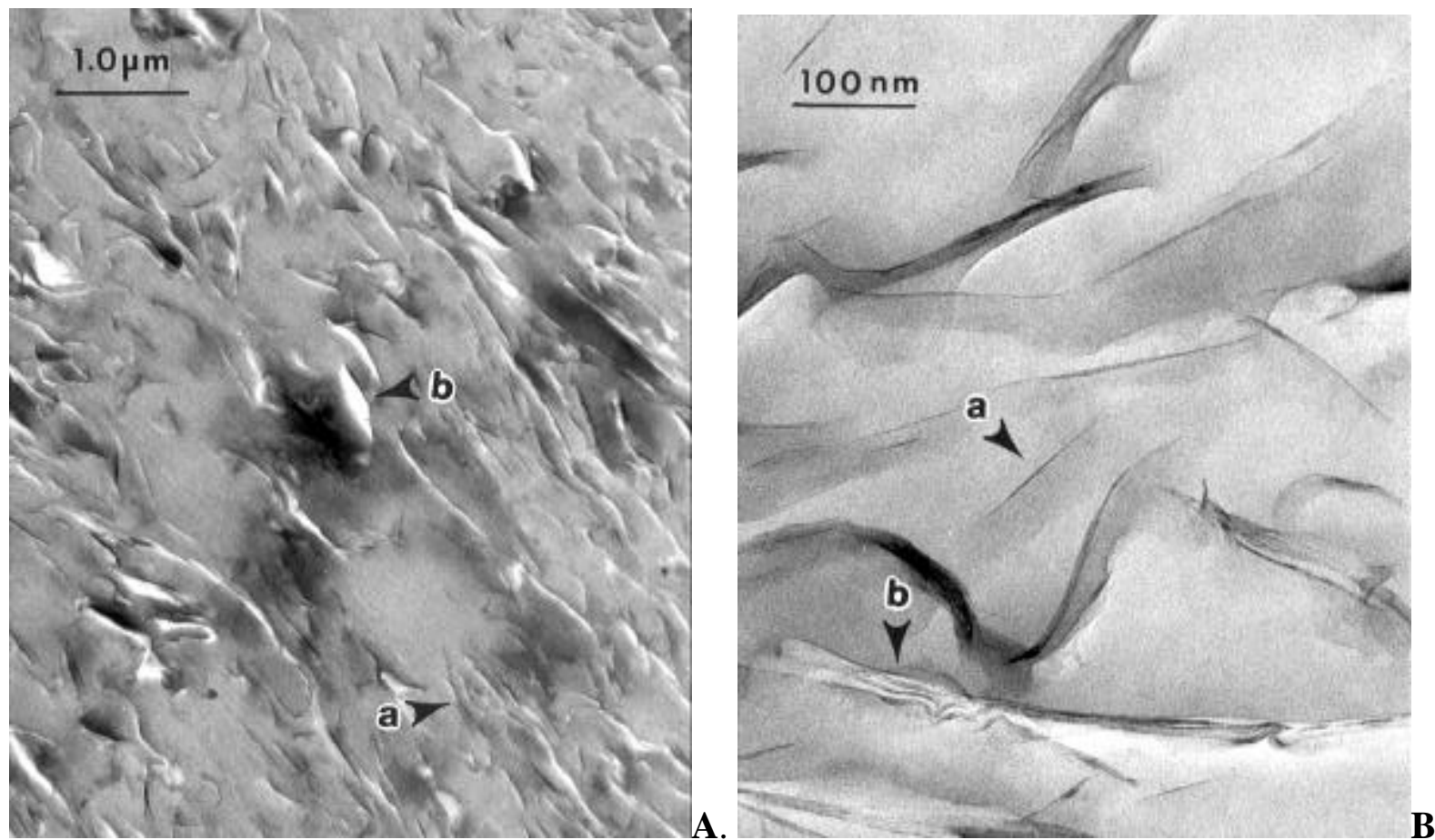

Figure 9. (A) Low magnification picture (14000x) of Polymer 1 + 10wt\% MMT-C12. The darker lines (a) are clay and the white areas (b) are holes in the TEM section.

Figure 10. (B) Medium magnification picture (140000x) of Polymer $1+10 \mathrm{wt} \%$ MMT-C12. Note single layers present (a) as well as some multi-layer tactoids (b).

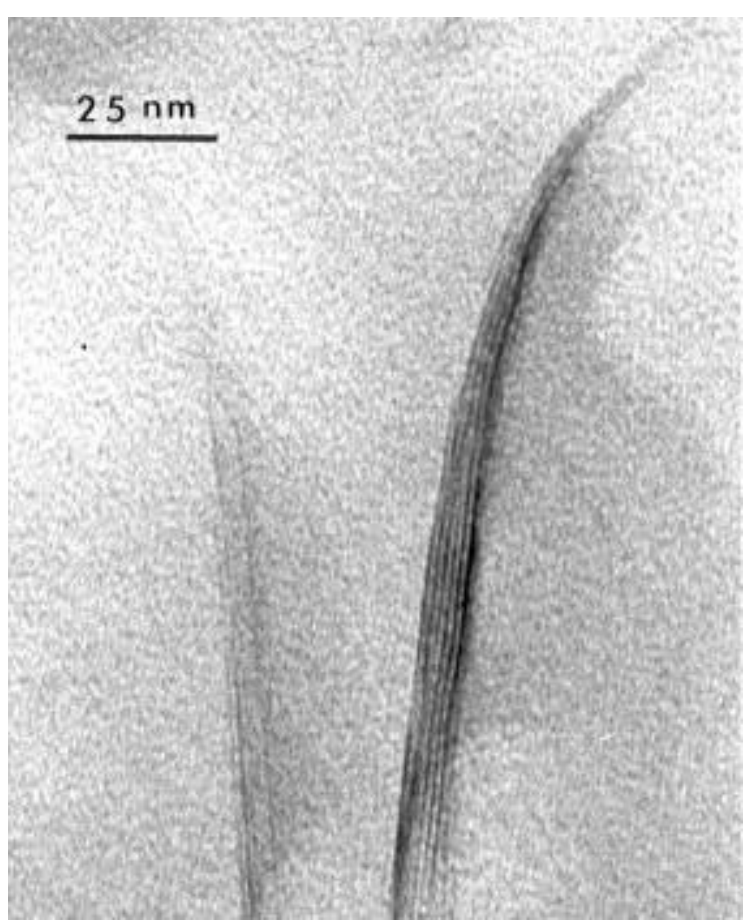

Figure 11. High magnification picture (600000x) of Polymer $1+10 \mathrm{wt} \%$ MMT-C12 showing a multi-layer tactoid. 

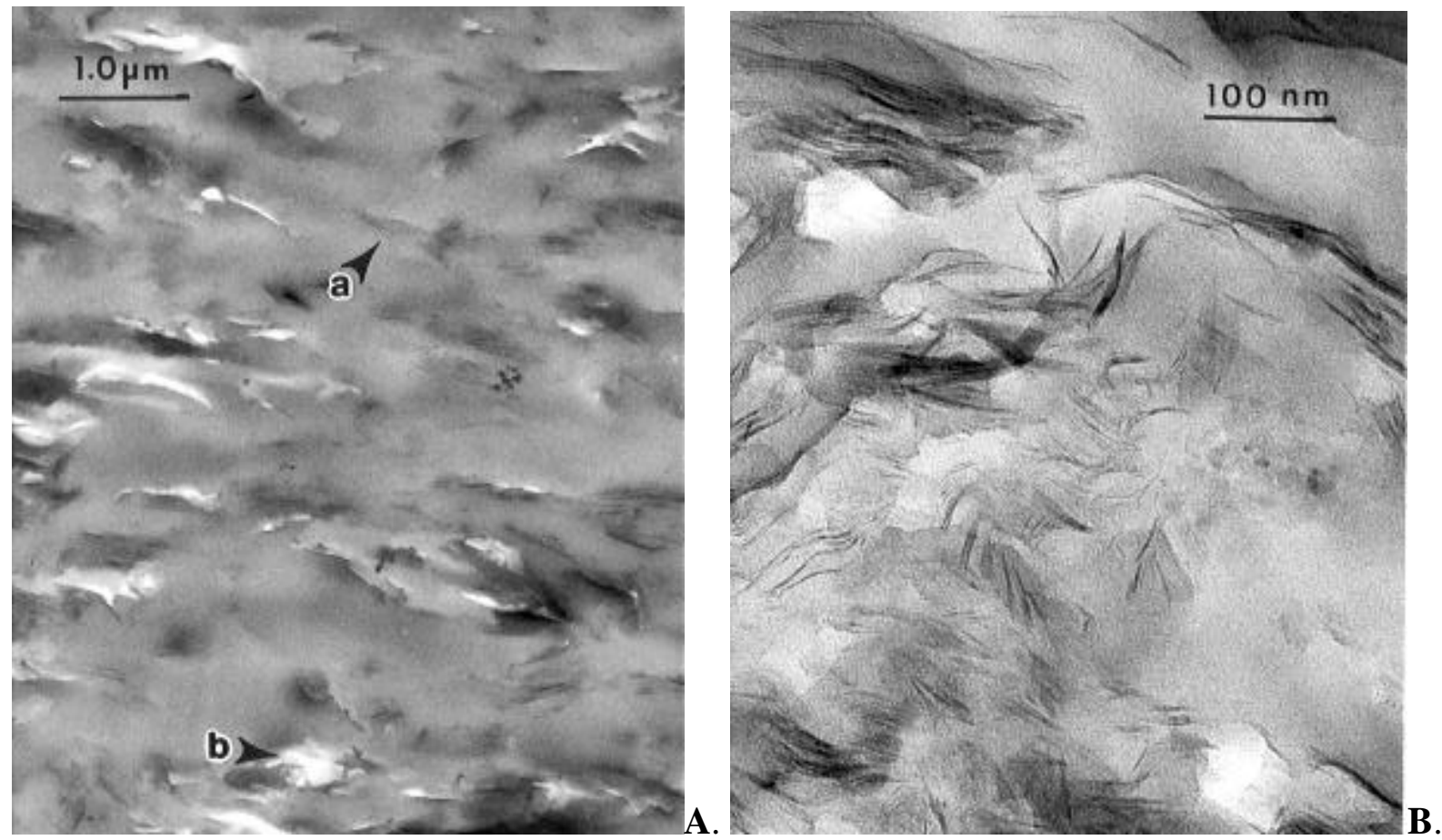

Figure 12. (A) Low magnification picture (14000x) of Polymer $1+10 \mathrm{wt} \%$ MMT-C12Acid. The small darker lines (a) are clay and the open white regions (b) are holes in the TEM section.

Figure 13. (B) Medium magnification picture (140000x) of Polymer 1 + 10wt\% MMT-C12Acid. This is a domain with many multi-layer intercalated tactoids.

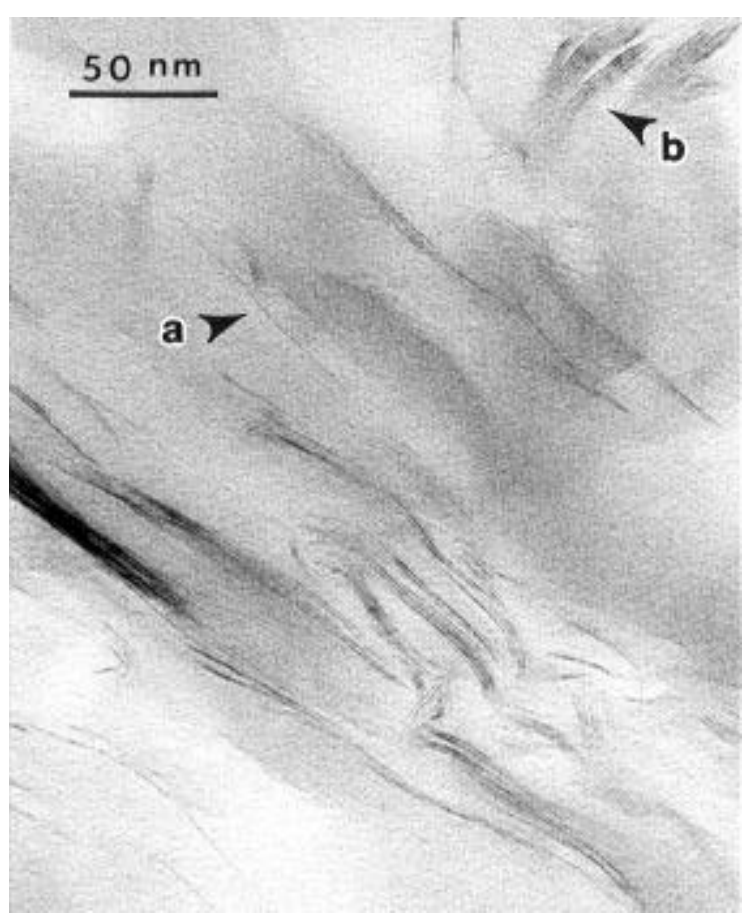

Figure 14. High magnification picture (300000x) of Polymer $1+10 w t \%$ MMT-C12Acid. Some single layers (a) are present as well as multi-layer intercalated tactoids (b). 


\subsection{Conclusions on the Synthesis of Polyetherimide-Clay Nanocomposites.}

We were able to successfully synthesize a PEI-nanocomposite using MMT-C12Acid via an in situ approach. However, this nanocomposite did not have the improved thermal stability we had hoped to achieve via this route. To evaluate the flammability properties of this nanocomposite using the cone calorimeter we will need to prepare larger quantities of this material. The synthetic route used to make this nanocomposite has its drawbacks. The first drawback is due to the low decomposition temperature of the organic treatment on the clay. Secondly, the oxidative instability of the diamine may have produced a polymer which had several defects in the polymer backbone. These two drawbacks most likely led to the lower than expected char yields and lower thermal stability relative to pure PEI 1. Further, the thermally unstable organic treatment on the clay may also have contributed to the low char yield. We are currently investigating new high temperature organic treatments for clays, which may allow the more commercially friendly approach of thermoplastic blending to be used in the preparation of a polyetherimide nanocomposite. We will also look at other polyetherimides other than polymer 1 which may lend themselves to easier synthetic routes. 


\subsection{THERMALLY INDUCED CHANGES IN THE CONDENSED PHASE OF BURNING POLYMERS}

\subsection{Introduction}

On the basis of evidence obtained in previous FAA sponsored investigations conducted in this laboratory, it now appears that the transport of volatile thermal degradation products through the condensed phase (as distinguished from the chemistry involved in producing these compounds) is the critical factor that determines the flammability of polymer/clay nanocomposites and other materials under consideration for future use in aircraft cabins. Thus, our focus this year was on using both computational and experimental tools to explore the changes that occur in the condensed phase when polymers are burned.

\subsection{Experimental.}

The fiber optic set-up used in the experiments consisted of a sapphire probe $(300 \mu \mathrm{m}$ in diameter and $10 \mathrm{~cm}$ in length) which was mounted on a steel rod and connected at both ends to zirconium fluoride $(\mathrm{ZrF})$ cables. These cables were connected to focusing optics, consisting of a pair of off-axis parabolic mirrors, that launch an evanescent wave at the source and capture it at the detector. A reflectance spectrum results from the attenuation of the evanescent wave due to absorption of the polymer (and its degradation products) in the immediate vicinity of the probe. Disk shaped samples (approximately $7.5 \mathrm{~cm}$ in diameter) of the polymers were placed on top of the probe which fit snugly into a groove that had been previously milled to facilitate a good contact between the polymer melt and the optical fiber. Although the sapphire probe can withstand temperatures up to $2000{ }^{\circ} \mathrm{C}$, the $\mathrm{ZrF}$ cables are sensitive to heat and had to be protected by water-cooled jackets constructed by winding plastic tubing around a copper pipe. As an added protection, these jackets were also wrapped in aluminum foil.

\subsection{Results}

\subsubsection{Computational Results}

A strategy for evaluating molecular weight distributions (which should enable us to calculate the melt viscosity during burning) from molecular dynamics (MD) simulations was developed based on a simple differential equation for the time dependence of the number average degree of polymerization, $\mathrm{x}$, derived by Boyd. The relevant equation is

$$
\frac{d x}{d t}=-\left(k_{i}+k_{I} \bar{R}\right) x^{2}
$$

where $\mathrm{k}_{\mathrm{i}}$ and $\mathrm{k}_{\mathrm{I}}$ are the rate constants for random scission and intermolecular hydrogen transfer and $\bar{R}$ is the steady-state concentration of free radicals. On the basis of previous results ${ }^{8}$, which are summarized in Figure 15, the Ahrrenius constants for $k_{i}$ in polypropylene are $E_{a}=323 \pm 25$ $\mathrm{kJ} / \mathrm{mol}$ and $\mathrm{A}=1.5 \times 10^{15} \mathrm{~s}^{-1}$. These values are in line with experimental values for bond scission reactions in small gas phase molecules and are consistent with TGA measurements of the global rate of mass loss during the thermal degradation of polypropylene. We are now in the process of performing additional simulations to obtain values for $\mathrm{k}_{\mathrm{I}}$. 


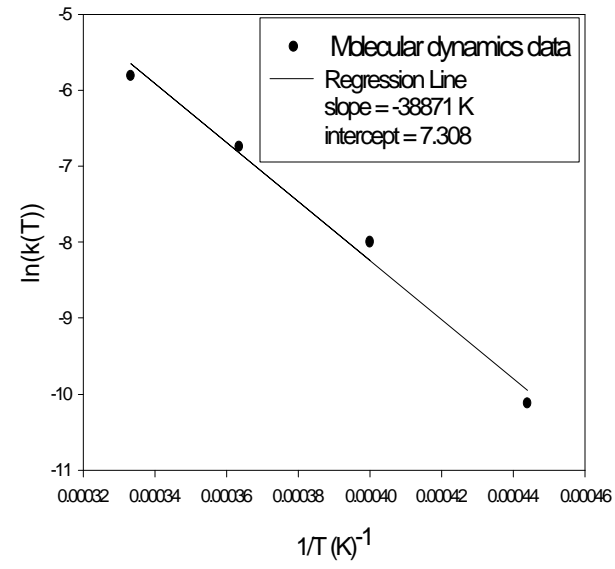

Random Scission Reactions

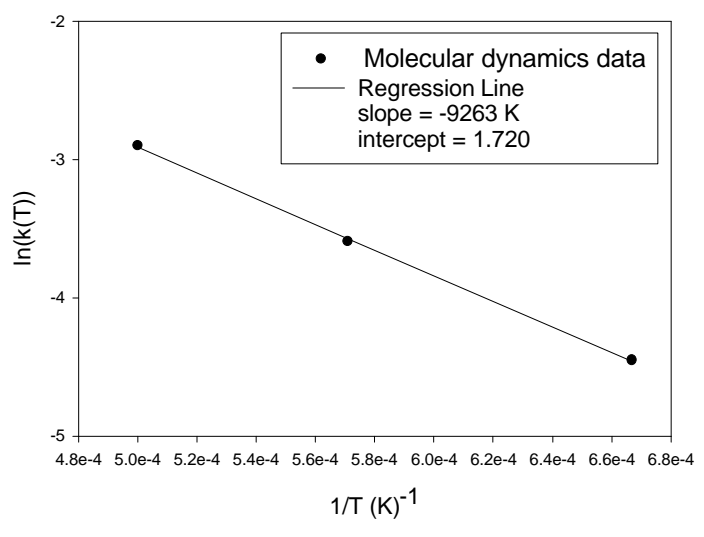

Propagation Reactions

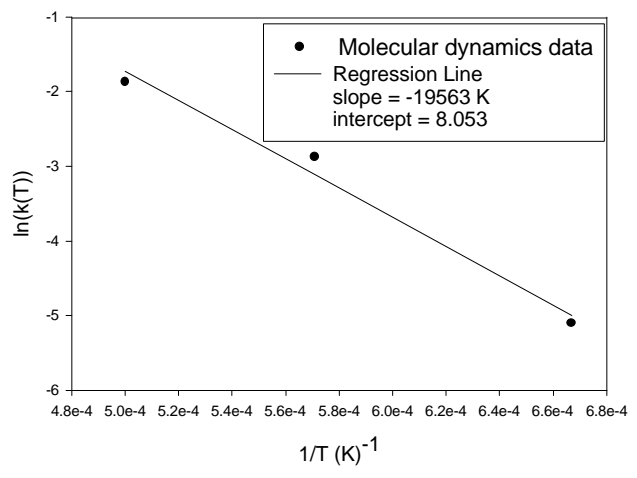

Termination Reactions

\section{$\frac{1}{m(t)} \frac{d m(t)}{d t}=-5.3 \times 10^{13} \exp \left(-\frac{237528}{R T}\right)$}

Experimental Values from Bockhorn et. al., Journ Anal. and Appl. Pyrolysis 46, (1998), 1-13.

$\mathrm{E}_{\mathrm{a}}=220000 \mathrm{~J} / \mathrm{mol}$ $\mathrm{A}=1.9 \times 10^{13} \mathrm{~s}^{-1}$

Figure 15. Computational data for polymer decompostions.

\subsubsection{Condensed Phase Infrared Absorbance Results.}

Condensed phase absorbance spectra of nylon- 6 and a nanocomposite of nylon- 6 and montmorillonite clay were acquired using a Midac Illuminator spectrometer ${ }^{\ddagger}$ during flammability measurements on the Cone Calorimeter. The nylon-6 and nylon-6/clay nanocomposite (mass fraction clay, $5 \%$ ) were obtained from UBE industries and used as received. The samples were exposed to a radiant heat flux of $35 \mathrm{~kW} / \mathrm{m}^{2}$ and ignited when there was sufficient volatilization to sustain gas phase combustion. Spectra were measured continuously until the flames selfextinguished, which was about 15 minutes (from the time the samples were exposed to the radiant heat) for the pure polymer and almost twice as long for the nanocomposite. A photograph showing the probe assembly and the burning polymer during an experiment is presented in figure 16.

The spectral window of the measurements was limited to the region extending from about $2250 \mathrm{~cm}^{-1}$ to $4000 \mathrm{~cm}^{-1}$ due to the transmission properties of the fiber optics. This frequency range encompasses absorption due to $\mathrm{C}-\mathrm{H}, \mathrm{N}-\mathrm{H}$, and $\mathrm{O}-\mathrm{H}$ stretching modes, which are active in 
the amines, amides and carboxylic acids produced in the thermal decomposition of nylon polymers ${ }^{9}$. Each spectrum was signal-averaged by co-adding 256 scans over the specified frequency range. This corresponds to a time resolution of about $30 \mathrm{~s}$ per spectrum at a frequency resolution of $4 \mathrm{~cm}^{-1}$.
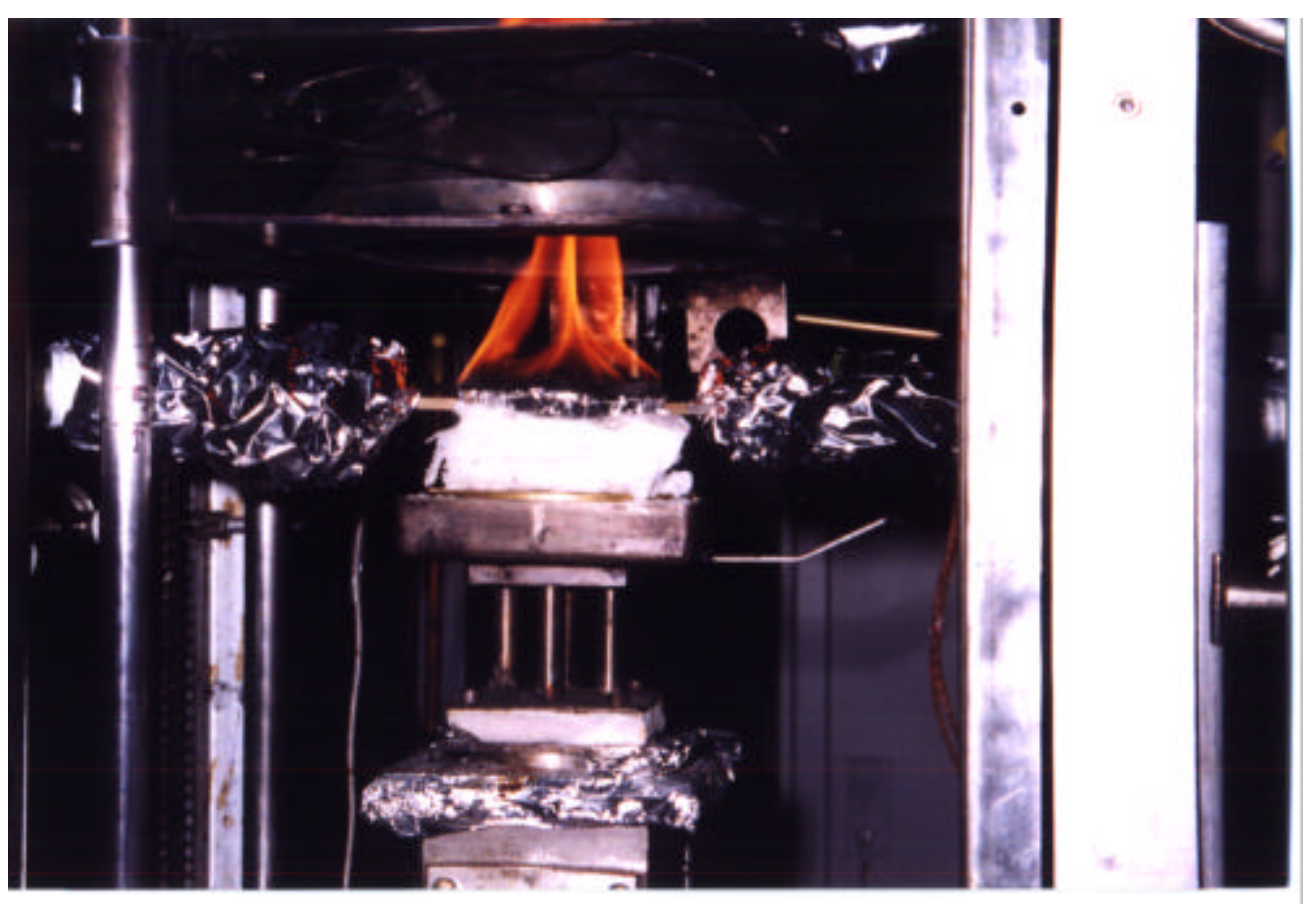

Figure 16. Experimental set-up showing the fiber optic probe imbedded in the burning polymer.

The spectra obtained early in the Cone Calorimeter experiment (i.e., before ignition) and in the vicinity of the peak HRR are compared to a spectrum of nylon- 6 measured using a diamond reflectance probe at room temperature in figure 17. These spectra are similar, but not identical, to spectra obtained from solid residues collected at various stages in the thermal decomposition of nylon- 6 carried out on a thermogravimetric analyser. ${ }^{10}$ The spectrum measured before ignition closely resembles the reference spectrum of nylon- 6 except for an apparent broadening of the peak centered at $3295 \mathrm{~cm}^{-1}$, which is probably an effect of the elevated temperature. The reference spectrum was measured at about $25^{\circ} \mathrm{C}$, whereas the temperature of the melt at this point in the Cone Calorimeter experiment was probably in excess of $300{ }^{\circ} \mathrm{C}$. The unresolved absorbance in the region between $3050 \mathrm{~cm}^{-1}$ and $3200 \mathrm{~cm}^{-1}$, which is evident in both the reference spectrum and in the spectrum prior to ignition, is probably due to the monomer $(\varepsilon-$ caprolactam) and its cyclic oligomers which have peaks centered at about $3055 \mathrm{~cm}^{-1}$ and 3185 $\mathrm{cm}^{-1}$. There is also an overtone of the amide II band in the spectrum of nylon- 6 at about 3085 $\mathrm{cm}^{-1}$ which is sensitive to the conformation of the polymer chains. ${ }^{11}$ 


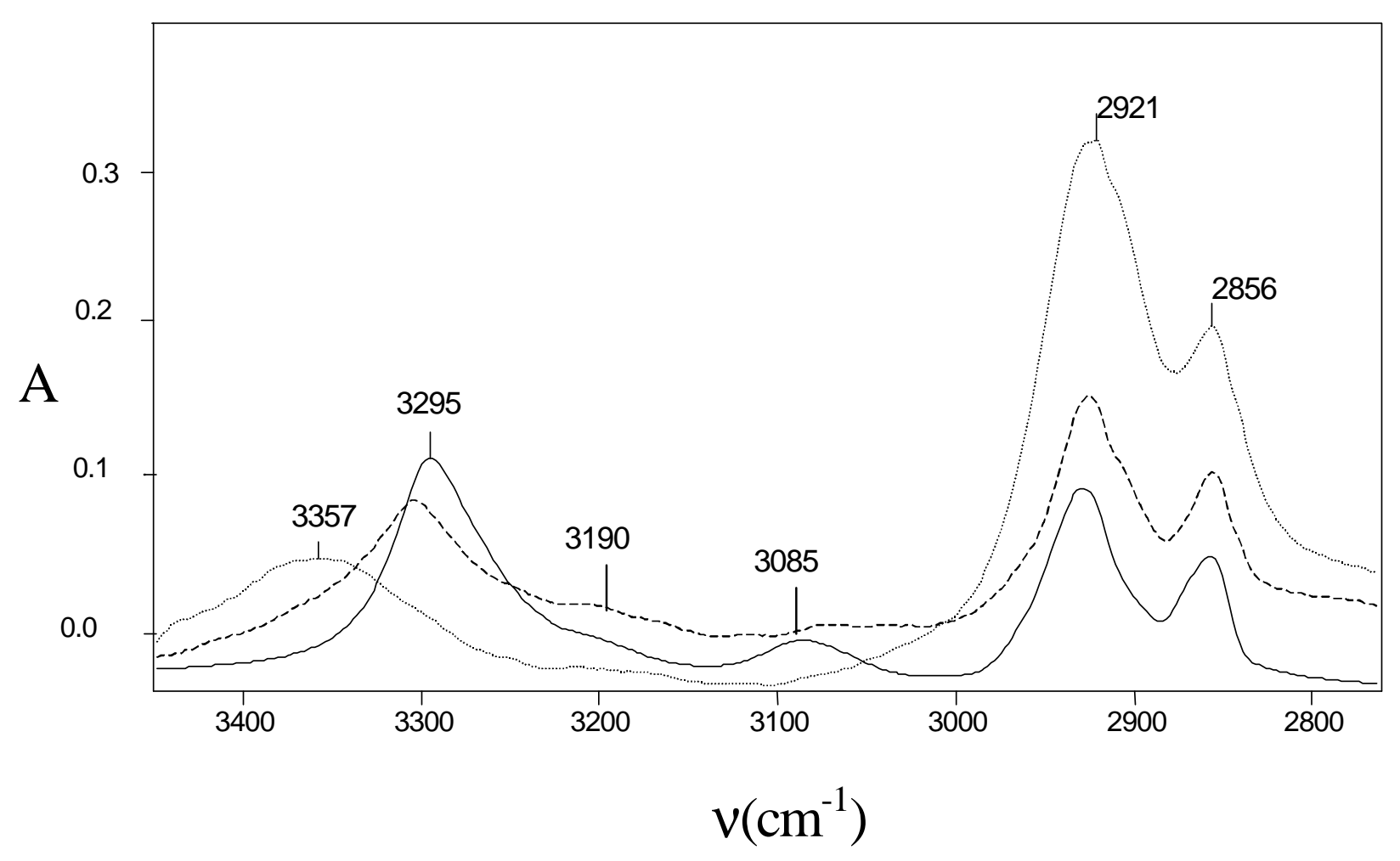

Figure 17. The spectra obtained from nylon-6 just before ignition (dashed line) and in the vicinity of the peak HRR (dotted line) are compared to a room temperature spectrum.

There are some obvious differences between the spectrum obtained in the vicinity of the peak in the HRR and the spectrum measured before ignition. In particular, there is a shift in the center of the N-H stretching vibration from about $3300 \mathrm{~cm}^{-1}$ to $3357 \mathrm{~cm}^{-1}$. This is consistent with spectral features that have been attributed to conformational changes resulting in the disruption of hydrogen bonds. ${ }^{12}$ In this case, the hydrogen bonds involve the carbonyl oxygen and amide hydrogen on adjacent anti-parallel chains. ${ }^{13}$ There is also a shoulder extending from about 3400 $\mathrm{cm}^{-1}$ to $3450 \mathrm{~cm}^{-1}$ that may be due to the formation of carboxylic end-groups resulting from the hydrolysis of peptide bonds in the interior of the polymer chains.

The spectra obtained from nylon- 6 and the nylon-6/clay nanocomposite, which were both measured about 4 minutes into the burn (after ignition but before the peak HRR), are compared in figure 18. The spectrum from the nanocomposite, although noisier, presumably because the melt did not adhere as well to the sapphire probe, exhibits features similar to those observed in the spectrum of the pure polymer. This observation is consistent with previous results from computer simulations ${ }^{14}$ and electron microscopy ${ }^{15}$ which indicate that the same products are produced during the thermal degradation of both materials, but, in the case of the nanocomposite, they become trapped in a char-like residue. This hypothesis will be examined further in future experiments, where both the condensed and gas phase spectra corresponding to nylon- 6 and the nylon-6/clay nanocomposite will be monitored at critical points along their HRR curves. 


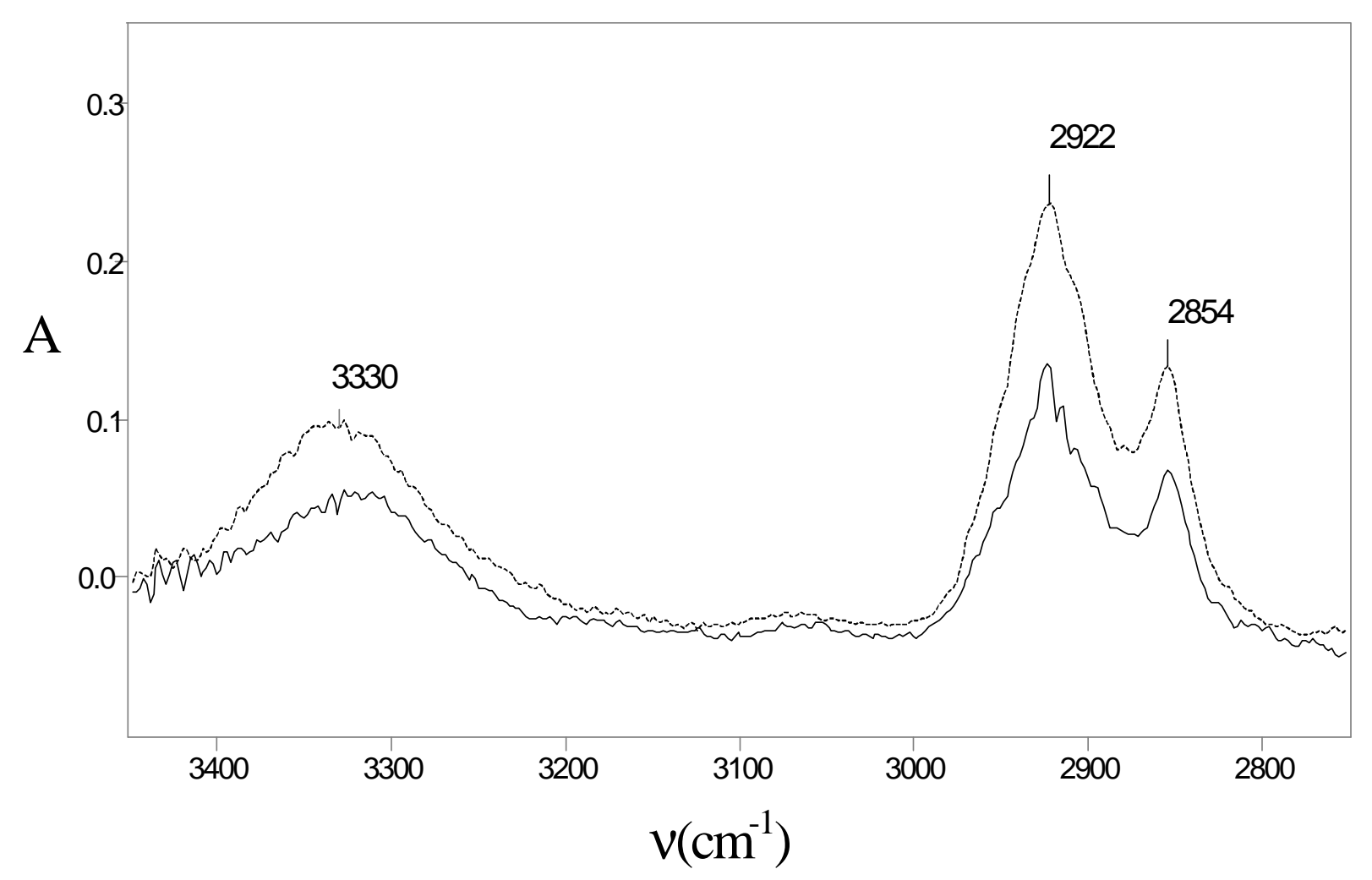

Figure 18. The spectrum from nylon-6 (dashed line), which was measured just after ignition, is compared to the spectrum of the nylon-6/clay nanocomposite (solid line) measured about the same time into the burn.

\subsection{Summary and Conclusions on Thermally Induced Changes in the Condensed Phase of Burning Polymers.}

Our computer program for simulating the thermal degradation of polymers was validated by comparing predicted values for the Ahrrenius constants associated with the thermal degradation of polypropylene to experimental values obtained from thermal gravimetric analysis. This approach to the prediction of the global rate of mass-loss from thermally degrading polymers was formally extended to provide a description of thermally induced changes in the molecular weight distribution of the polymer melt. This will facilitate a better understanding of the transport phenomena that are critical in determining the flammability of aircraft materials.

Changes in the condensed phase mid-infrared (MIR) spectra of nylon-6 and a nylon6/clay nanocomposite were monitored in real-time during flammability measurements conducted on the Cone Calorimeter. The spectra obtained during the burning process were of sufficiently high quality to show the progression of the material in contact with the probe from molten polymer to thermal degradation products. The spectral features are consistent with the increasing formation of $\varepsilon$-caprolactam as a result of depolymerization reactions. On the basis of these results, we have concluded that the use of MIR fiber optics in conjunction with a fourier transform infrared (FTIR) spectrometer to monitor changes in the condensed phase chemistry of burning polymers is both feasible and useful. In the future, we hope to use this tool to in conjunction with the molecular modeling effort to identify major degradation products of 
burning polymers and thereby to elucidate the chemistry responsible for the heat release at critical points along the corresponding HRR curves.

\section{Acknowledgments}

The authors wish to thank the Federal Aviation Administration under interagency agreement No. DTFA03-99-X-9009. We also wish to thank General Electric Corporation for the gift of bisphenol A dianhydride and polymer 1 (Ultem ${ }^{\mathrm{TM}}$ ). We also wish to thank Southern Clay Products for the donation of montmorillonite clays; Na-MMT, MMT-C12, and MMT-C12Acid. Finally we wish to thank Dr. James Cline of NIST for use of XRD facilities.

\section{Future Work}

While we were able to synthesize a polyetherimide nanocomposite, the resulting thermal properties were not improved. We need to scale up the preparation of PEI 1-nanocomposite to evaluate its flammability in the Cone Calorimeter. However, the preparation of more material is limited by the synthetic routes available and by the organic treatment on the clay. We believe that the weakest part of trying to make a polyetherimide nanocomposite is the organic treatment on the clay. Most ammonium organic treatments are rarely stable beyond $250{ }^{\circ} \mathrm{C}$. This limits their use to polymers which can be processed below $250{ }^{\circ} \mathrm{C}$. Therefore making nanocomposites of thermoplastic engineering plastics such as Ultem (1) or polycarbonate is very difficult to accomplish. We had hoped that the solvent blending approach would work, but due to the limited solubility of Ultem this approach failed.

Since the organic treatment is crucial to the successful preparation of a polymer-clay nanocomposite, we are currently looking into different types of organic treatments with thermal stabilities above $250{ }^{\circ} \mathrm{C}$. One treatment currently under investigation is to use 18-crown-6 (2), (Figure 19) which can complex with the sodium cations on sodium montmorillonite (Na-MMT). 18-Crown-6 is more thermally stable than ammonium cations, and further, it can be used on NaMMT without removing the sodium cations. Na-MMT is the most thermally stable version of montmorillonite, so to try and leave it intact by complexing organophilic ligands to the sodium cations may be an approach which would yield success.

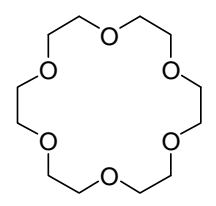

2

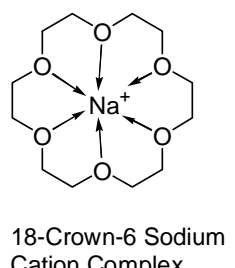

Cation Complex

Figure 19. 18-Crown-6 and 18-Crown-6 sodium cation $\left(\mathrm{Na}^{+}\right)$complex.

We will also be collaborating with NASA-Langley on the preparation of polyetherimide nanocomposites. Specifically, we will be using a NASA synthesized polyetherimide, LARC TPI. NASA Langley facilities has prepared experimental resins which have high thermal stability, but low melting (processing) temperatures. This will allow the synthesis of nanocomposites which can be prepared by melt blending methods rather than the more difficult in situ techniques. 
We plan to continue our work on the development and validation of MD_REACT ${ }^{8}$ modeling software with a focus on further refining our capability to use this model to simulate thermally induced changes in the condensed phase of burning polymers.

\section{References}

(1) Lyon, R. E. "Memorandum: FAA Advanced Fire-Safe Aircraft Materials Research Program.” 1994, p. 1.

(2) (a) Gianellis, E. P. Advanced Materials 1996, 8, 29. (b) Kojima, Y.; Usuki, A.; Kawasumi, M.; Okada, A.; Fukushima, Y.; Kurauchi, T.; Kamigaito, O. J. Mater. Res. 1993, 8, 1185. (c) Wang, Z.; Pinnavaia, T. J. Chem. Mater. 1998, 10, 1820. (d) Burnside, S. D.; Giannelis, E. P. Chem. Mater. 1995, 7, 1597. (e) Lee, J.; Takekoshi, T.; Giannelis, E. P. Mat. Res. Soc. Symp. Proc. 1997, 457, 513.

(3) (a) Gilman, J. W.; Kashiwagi, T.; Lichtenhan, J. D. SAMPE Journal 1997, 33, 40. (b) Gilman, J. W.; Kashiwagi, T.; Brown, J. E. T.; Lomakin, S.; Giannelis, E. P.; Manias, E. Proceedings of 43rd Inter. SAMPE Symp. And Exib. 1998, 1053. (c) Gilman, J. W.; Kashiwagi, T.; Nyden, M.; Brown, J. E. T.; Jackson, C. L.; Lomakin, S.; Giannelis, E. P.; Manias, E. Chemistry and Technology of Polymer Additives. The Royal Society of Chemistry, Cambridge, 1999, 249-265. (d) Gilman, J. W. App. Clay Sci. 1999, 15, 31.

(4) (a) Lan, T.; Kaviratna, P. D.; Pinnavaia, T. J. Chem. Mater. 1994, 6, 573. (b) Tyan, H.; Liu, Y.; Wei, K. Chem. Mater. 1999, 11, 1942. (c) Tyan, H.; Liu, Y.; Wei, K. Polymer 1999, 40, 4877.

(5) (a) Vaia, R. A.; Ishii, H.; Giannelis, E. P. Chem. Mater. 1993, 5, 1694. (b) Vaia, R. A.; Vasudevin, S.; Krawiec, W.; Scanlon, L. G.; Giannelis, E. P. Adv. Mater. 1995, 7, 154. (c) Giannelis, E. P. Adv. Mater. 1996, 8, 29.

(6) (a) Yano, K.; Usuki, A.; Okada, A.; Kurauchi, T.; Kamigaito, O. J. Poly. Sci, Part A 1993, 31, 2493. (b) Yang, Y.; Zhu, Z.; Yin, J.; Wang, X.; Qi, Z. Polymer 1999, 40, 4407.

(7) This industrial technique is used by General Electric Inc. to make Ultem (polymer 1). The monomers for this synthetic route are not commercially available and the nuances of the chemistry are best known to GE.

(8) Nyden, M. R., International Aircraft Fire and Cabin Safety Research Conference, November 16-20, Atlantic City, NJ (1998).

(9) Levchik, S. V.; Weil, E. D.; Lewin, M. Polym. Int. 1999, 48, 1.

(10) Levchik, S. V.; Costa, L.; Camino, G. Polym. Degrad. and Stab. 1992, 36, 229.

(11) Silverstein, R. M; Bassler, G. C.; Morrill, T. C. Spectrometric Identification of Organic Compounds, (John Wiley and Sons, NY, 1963), Third Edition, p. 108.

(12) de Haseth, J. A.; Andrews, J. E.; McClusky, J. V.; Priester, R. D. Jr.; Harthcock, M. A.; Davis, B. L. Appl. Spectrosc. 1993, 47, 173.

(13) Holmes, D. R.; Bunn, C. W.; Smith, D. J. J. Polym. Sci., 1955, 17, 159.

(14) Nyden, M. R.; Gilman, J.W. Computational and Theoretical Polymer Science 1997, 7, 191.

(15) Gilman, J. W.; Kashiwagi, T.; Nyden, M. R.; Brown, J. E. T.; Jackson, C. L.; Lomakin, S.; Giannelis, E. P.; Manias, E. "Flammability Studies of Polymer Layered Silicate Nanocomposites: Polyolefin, Epoxy, and Vinyl Ester Resins," in: Chemistry and Technology of Polymer Additives, S. Al-Malaika, A. Golovoy, and C.A. Wilkie, Ed., (Blackwell Science Ltd., Oxford, 1999). 